\title{
Paris-rue-Madame database: a 3D mobile laser scanner dataset for benchmarking urban detection, segmentation and classification methods
}

\author{
Andrés Serna ${ }^{1}$, Beatriz Marcotegui ${ }^{1}$, François Goulette ${ }^{2}$ and Jean-Emmanuel Deschaud ${ }^{2}$ \\ ${ }^{1}$ MINES ParisTech, CMM-Center for mathematical morphology, 35 rue St Honoré, 77305 Fontainebleau, France \\ ${ }^{2}$ MINES ParisTech, CAOR-Center for robotics, 60 boulevard Saint-Michel, 75272 Paris, France \\ \{andres.serna_morales, beatriz.marcotegui, francois.goulette, jean-emmanuel.deschaud\}@mines-paristech.fr
}

\begin{abstract}
Keywords: 3D database, mobile laser scanner, urban analysis, segmentation, classification, point-wise evaluation.
Abstract: $\quad$ This paper describes a publicly available 3D database from the rue Madame, a street in the $6^{\text {th }}$ Parisian district. Data have been acquired by the Mobile Laser Scanning (MLS) system L3D2 and correspond to a $160 \mathrm{~m}$ long street section. Annotation has been carried out in a manually assisted way. An initial annotation is obtained using an automatic segmentation algorithm. Then, a manual refinement is done and a label is assigned to each segmented object. Finally, a class is also manually assigned to each object. Available classes include facades, ground, cars, motorcycles, pedestrians, traffic signs, among others. The result is a list of $(X, Y, Z$, reflectance, label, class) points. Our aim is to offer, to the scientific community, a 3D manually labeled dataset for detection, segmentation and classification benchmarking. With respect to other databases available in the state of the art, this dataset has been exhaustively annotated in order to include all available objects and to allow point-wise comparison.
\end{abstract}

\section{INTRODUCTION}

Nowadays, LiDAR technology ("light detection and ranging") has been prospering in the remote sensing community thanks to developments such as: Aerial Laser Scanning (ALS), useful for large scale buildings survey, roads and forests; Terrestrial Laser Scanning (TLS), for more detailed but slower urban surveys in outdoor and indoor environments; Mobile Laser Scanning (MLS), less precise than TLS but much more productive since the sensors are mounted on a vehicle; and more recently, "stop and go" systems, easy transportable TLS systems making a trade off between precision and productivity.

Thanks to all these technologies, the amount of available 3D geographical data and processing techniques has bloomed in recent years. Many semiautomatic and automatic methods aiming at analyzing 3D urban point clouds can be found in the literature. It is an active research area. However, there is not a general consensus about the best detection, segmentation and classification methods. This choice is application dependent. One of the main drawbacks is the lack of publicly available databases allowing benchmarking.

In the literature, most available urban data consist in close-range images, aerial images, satellite images but a few laser datasets (ISPRS, 2013; IGN, 2013).
Moreover, manual annotations and algorithm outputs are rarely found in available 3D repositories (Nüchter and Lingemann, 2011; CoE LaSR, 2013).

Some available data include Oakland dataset (Munoz et al., 2009), which contains 1.6 million points collected around Carnegie Mellon University campus in Oakland, Pittsburgh, USA. Data are provided in ASCII format: (X, Y, Z, label, confidence) one point per line, vrml files and label counts. The training/validation and testing data contains 5 labels (scatter misc, default wires, utility poles, load bearing and facades). Ohio database (Golovinskiy et al., 2009) is a combination of ALS and TLS data in Ottawa city (Ohio, USA). It contains 26 tiles $100 \times$ 100 meters each with several objects such as buildings, trees, cars and lampposts. However, ground truth annotations only consists in a 2D labeled point in the center of each object. In that sense, segmentations results cannot be evaluated point by point. Enschede database (Zhou and Vosselman, 2012) contains residential streets approximatively $1 \mathrm{~km}$ long in the Enschede city (The Netherlands). Ground truth annotation consists in 2D geo-referenced lines marking curbstones. A well- defined evaluation method is available using buffers around each $2 \mathrm{D}$ line. The drawback of this dataset is that no other objects are annotated. Paris-rue-Soufflot database (Hernández 
and Marcotegui, 2009) contains MLS data from a street $500 \mathrm{~m}$ long in the $5^{\text {th }}$ Parisian district. Six classes have been annotated: facades, ground, cars, lampposts, pedestrians and others.

In this paper, we present a 3D MLS database for benchmarking detection, segmentation and classification methods. Each point in the 3D point cloud has been segmented and classified, allowing pointwise evaluations. Additionally, our annotation includes all available objects in the urban scene. Data have been acquired and processed in the framework of TerraMobilita project (http://cmm.ensmp.fr/ TerraMobilita/ ).

Paper organization is as follows. Section 2 reminds some basic definitions. Section 3 describes Paris-rue-Madame database. Section 4 explains the MLS system and acquisition details. Section 5 briefly presents our manually assisted annotation protocol. Finally, Section 7 concludes the work.

\section{BACKGROUND}

A typical 3D urban analysis method includes 5 main steps: i) data filtering/down-sampling in order to reduce outliers and redundant data; ii) Digital Terrain Model (DTM) generation; iii) object detection in order to define object hypotheses and regions of interest; iv) object segmentation in order to extract each individual object; and v) object classification in order to assign a semantic category to each object. In the scientific community several definitions can be found for detection, segmentation and classification steps. For clarity, let us define these concepts in the way we intend they should be understood with this dataset:

Detection: An object is considered detected if it is included in the list of object hypotheses, i.e. it has not been suppressed by any filtering/downsampling method and it has not been included as part of the DTM. Note that an object hypothesis may contain several connected objects or even contain only a part of an object. In the detection step, we are only interested in keeping all possible objects. This is important because in most works reported in the literature, non-detected objects cannot be recovered in subsequent algorithm steps.

Segmentation: An object is considered segmented if it is correctly isolated as a single object, i.e. connected objects are correctly separated (there is no sub-segmentation) and each individual object is entirely inside of only one connected component (there is no over-segmentation). This is important because many algorithms based on clustering and connected components can wrongly gather objects touching each other, e.g. motorcycles parked next to the facade, pedestrians walking together, cars closely parked to others, among others.

Classification: In the classification step, a category is assigned to each segmented object. Each class represents an urban semantic entity. Depending on the application, several classes can be defined: facade, ground, curbstone, pedestrian, car, lamppost, etc.

\section{DATA DESCRIPTION}

Paris-rue-Madame dataset contains 3D MLS data from rue Madame, a street in the $6^{\text {th }}$ Parisian district (France). Figure 1 shows an orthophoto from the test zone, approximatively a $160 \mathrm{~m}$ long street section between rue Mézières and rue Vaugirard. The acquisition was made on February 8, 2013 at 13:30 Universal Time (UT).

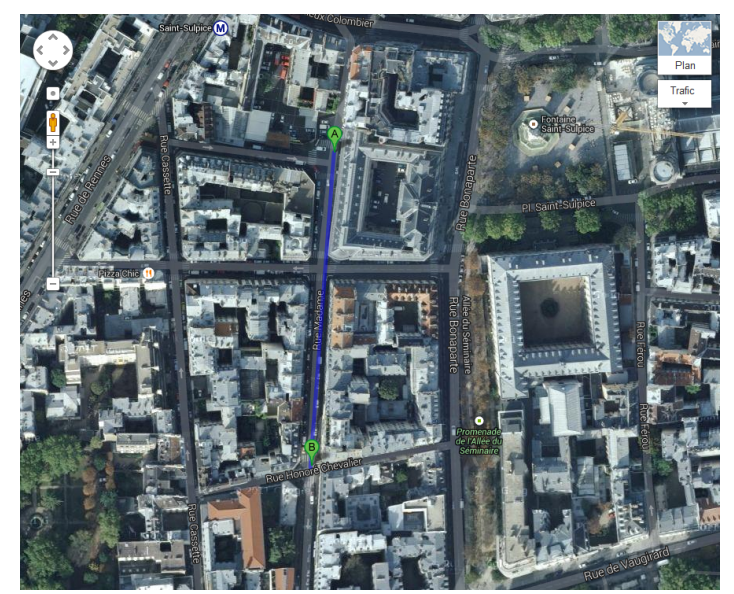

Figure 1: Rue Madame, Paris (France). Orthophoto from IGN-Google Maps.

The dataset contains two PLY files with 10 million points each. Each file contains a list of $(X, Y, Z$, reflectance, label, class) points, where $X Y Z$ correspond to geo-referenced $(E, N, U)$ coordinates in Lambert 93 and altitude IGN1969 (grid RAF09) reference system, reflectance is the laser intensity, label is the object identifier obtained after segmentation and class determines the object category. An offset has been subtracted from $X Y$ coordinates with the aim of increasing data precision: $X_{0}=650976 \mathrm{~m}$ and $Y_{0}=6861466 \mathrm{~m}$, respectively. The available files are "GT_Madame1_2.ply" and "GT_Madame1_3.ply", both of them coded as binary big endian version 1 . 
Figure 2 presents one of the 3D point clouds of this database colored by the point height ( $Z$ coordinate), the reflectance, the object label and the object class.

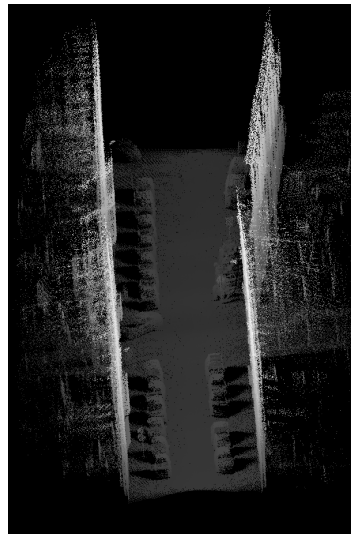

(a) Height ( $\mathrm{Z}$ coordinate)

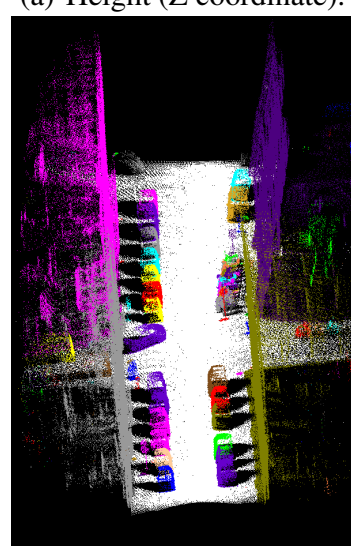

(c) Object label.

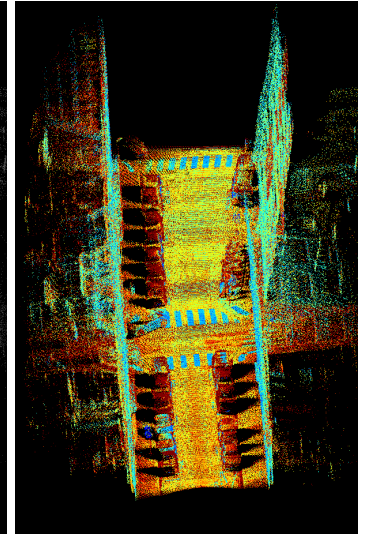

(b) Laser reflectance.

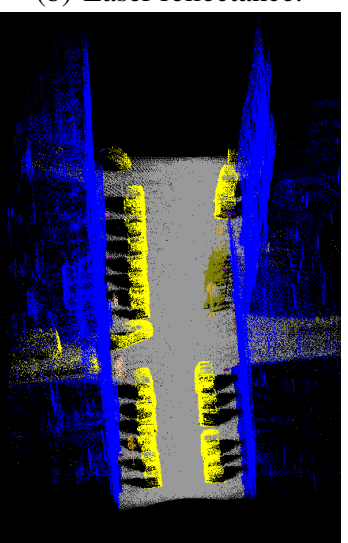

(d) Object class.
Figure 2: "GT_Madame1_2.ply" file: 3D point cloud colored by its available fields. For the object label, each color represents a different object (only for visualization purposes, some colors have been repeated). For object class visualization: facades (blue), ground (gray), cars (yellow), motorcycles (olive), traffic signs (goldenrod), pedestrians (pink).

This database contains 642 objects categorized in 26 classes, as shown in Table 1. It is noteworthy that several objects inside buildings have been acquired through windows and open doors, these objects have been annotated as facades. Other several "special" classes have been added because they are too different to be mixed with others. For instance, fast pedestrians and pedestrians+something have different geometrical features than a simple pedestrian. The idea of this annotation is including as much classes as possible, then each user may gather or exclude classes depending on the application.

It is noteworthy that this database is different from
Table 1: Available classes and number of objects in the Paris-Rue Madame dataset.

\begin{tabular}{llcc}
\hline & & \multicolumn{2}{c}{ Number of objects } \\
\hline Class & Class name & file 1_2 & file 1_3 \\
\hline 0 & Background & 7 & 35 \\
1 & Facade & 181 & 117 \\
2 & Ground & 4 & 23 \\
4 & Cars & 39 & 31 \\
7 & Light poles & 0 & 1 \\
9 & Pedestrians & 3 & 7 \\
10 & Motorcycles & 23 & 9 \\
14 & Traffic signs & 5 & 1 \\
15 & Trash can & 2 & 1 \\
19 & Wall Light & 6 & 1 \\
20 & Balcony Plant & 3 & 2 \\
21 & Parking meter & 1 & 1 \\
22 & Fast pedestrian & 2 & 2 \\
23 & Wall Sign & 1 & 3 \\
24 & Pedestrian + something & 1 & 0 \\
25 & Noise & 46 & 80 \\
26 & Pot plant & 0 & 4 \\
\hline \multicolumn{4}{r}{ Total } \\
\hline
\end{tabular}

others available in the state of the art since the entire $3 \mathrm{D}$ point cloud has been segmented and classified, i.e. each point contains a label and a class. Thus, pointwise evaluation of detection, segmentation and classification methods is possible.

\section{ACQUISITION}

The acquisition has been carried out by the MLS system L3D2 from the robotics laboratory CAORMINES ParisTech (Goulette et al., 2006). This system is equipped with a Velodyne HDL32, as shown in Figure 3. In this system, several lasers are mounted on upper and lower blocks of 32 lasers each and the entire unit spins, giving much denser point clouds than classic Riegl sensors (Velodyne, 2012).

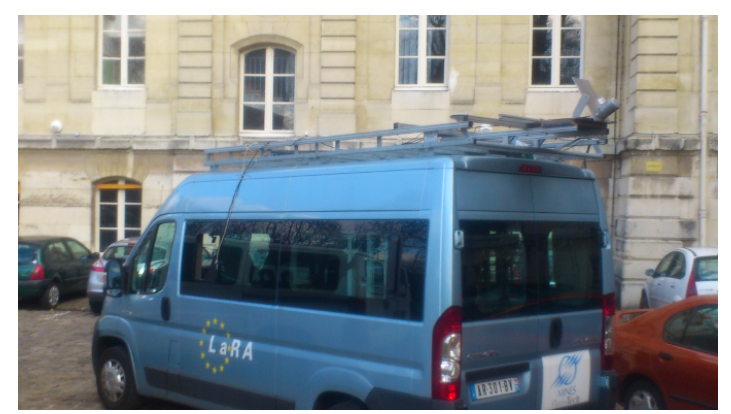

Figure 3: MLS system L3D2 from CAOR-MINES ParisTech 


\section{ANNOTATION}

Annotation has been carried out in a manually assisted way. An initial segmentation is obtained using an automatic method based on elevation images (Serna and Marcotegui, 2013b). The work-flow is shown in Figure 4. For further details and complete analyses in each step, the reader is also encouraged to review the following three works (Hernández and Marcotegui, 2009) (Serna and Marcotegui, 2013a) (Serna and Marcotegui, 2013c).

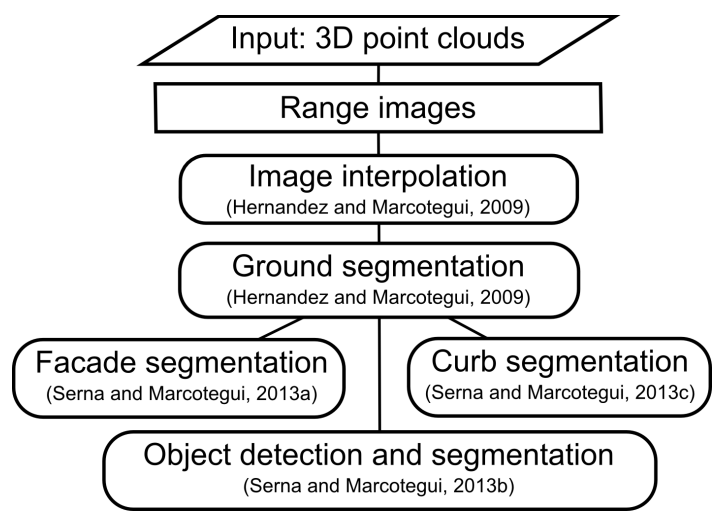

Figure 4: Work-flow of our automatic segmentation methodology.

After this automatic method, a manual refinement is carried out in order to correct possible errors in detection and segmentation steps. Then, a label is assigned to each segmented object. Finally, a class is also manually assigned in order to categorize each segmented object.

During manual refinement, three typical segmentation errors are both found and corrected: i) bad segmentation of some connected objects, e.g. motorcycles parked close to each other are not correctly separated, as shown in Figure 5; ii) over-segmentation of some objects due to artifacts or noise, i.e. some cars are over-segmented on their roof, as shown in Figure 6; iii) sub-segmentation of some objects, i.e. some objects touching the facades are not well separated, as shown in Figure 7.

\section{DOWNLOAD \& LICENSE}

Paris-rue-Madame database is avail-
able at: http://cmm.ensmp.fr/ serna/
rueMadameDataset.html and it is made avail-
able under the Creative Commons Attribution
Non-Commercial No Derivatives (CC-BY-NC-ND-
3.0) Licence.

(Cette œuvre est mise à disposition selon les termes

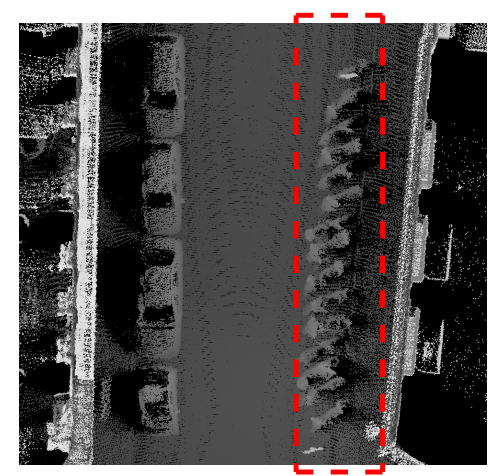

(a) Height

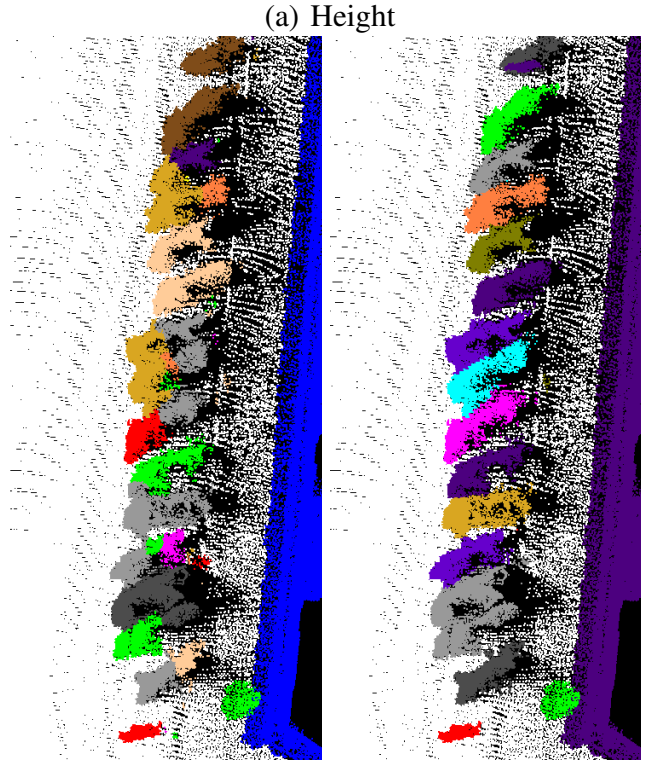

(b) Automatic segmen- (c) Manual refinement tation (zoomed) (zoomed)

Figure 5: Manual refinement of connected motorcycles.

de la Licence Creative Commons Attribution - Pas d'Utilisation Commerciale - Pas de Modification 3.0 France http://creativecommons.org/licenses/ by-nc-nd/3.0/fr/ ).

\section{CONCLUSIONS}

We have presented a 3D MLS database manually annotated from rue Madame, a street in the $6^{\text {th }}$ Parisian district. Each 3D point has been labeled and classified, resulting in a list of $(X, Y, Z$, reflectance, label, class) points.

The database has been acquired by the L3D2 vehicle, a MLS system from the Robotics laboratory (CAOR) at MINES ParisTech. A distinctive feature of this system is that it uses a Velodyne sensor aligned in a similar way to a Riegl sensor but providing much 


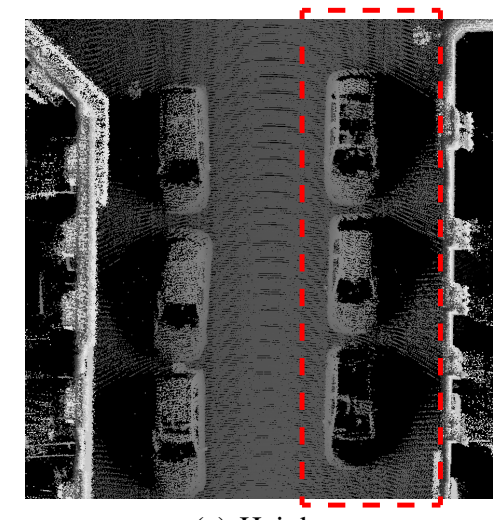

(a) Height
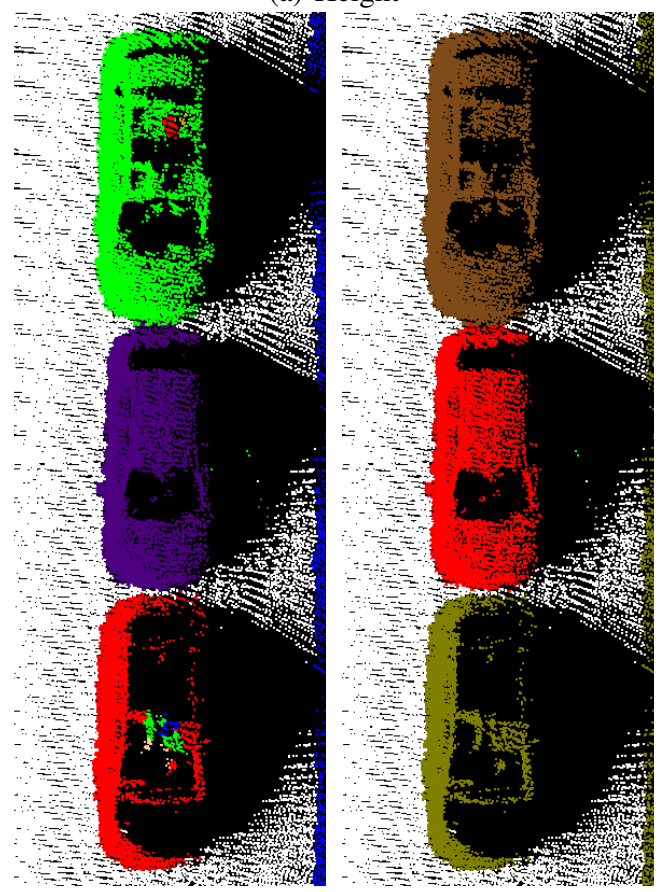

(b) Automatic segmenta- (c) Manual refinement tion (zoomed) (zoomed)

Figure 6: Manual refinement of over-segmented cars.

denser 3D point clouds.

Annotation has been carried out in a manual assisted way by the Center for mathematical morphology (CMM) at MINES ParisTech. First, an automatic segmentation method is applied. Then, manual refinement and classification are done. This approach is faster than a completely manual approach and it provides accurate results. This dataset is different from others available in the state of the art since each point has been segmented and classified, allowing pointwise benchmarking.

In future works, other datasets acquired in the framework of TerraMobilita project will be annotated and made available to the scientific community.
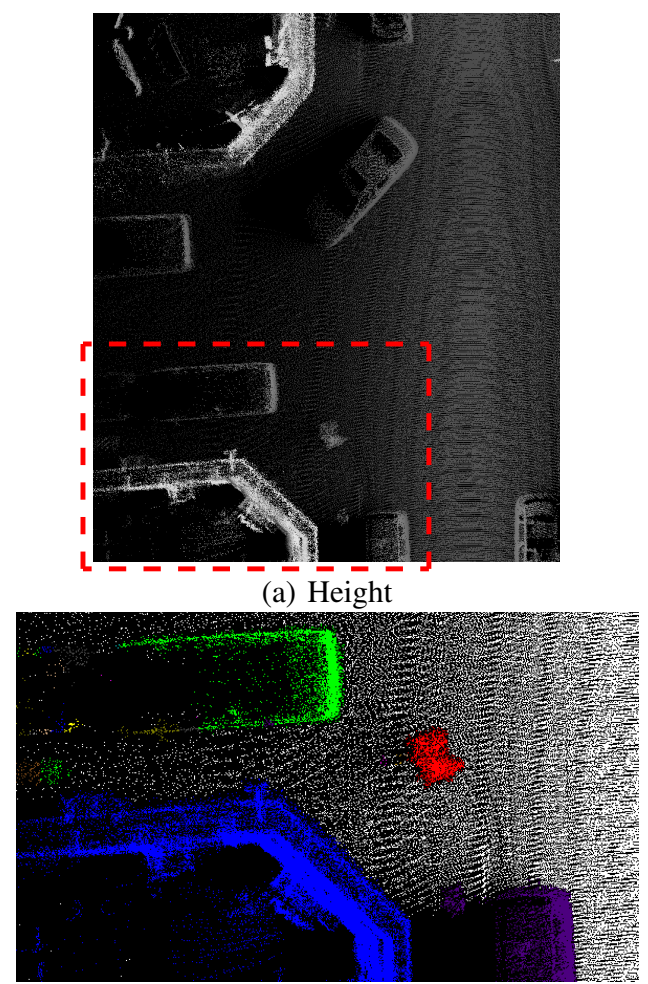

(b) Automatic segmentation (zoomed)

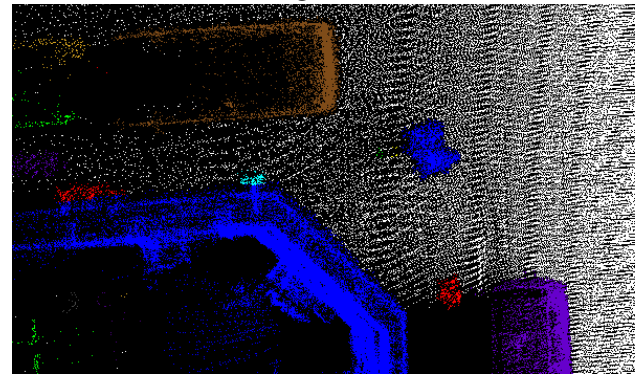

(c) Manual refinement (zoomed)

Figure 7: Manual refinement of sub-segmented objects touching the facade.

\section{ACKNOWLEDGEMENTS}

The work reported in this paper has been performed as part of Cap Digital Business Cluster TerraMobilita Project.

\section{REFERENCES}

CoE LaSR (2013). Centre of Excellence in Laser Scanning Research. Finnish Geodetic Institut. http: //www.fgi.fi/coelass/ (Last accessed: December $16,2013)$.

Golovinskiy, A., Kim, V., and Funkhouser, T. (2009). Shape-based recognition of 3D point clouds in urban 
environments. In IEEE International Conference on Computer Vision, pages 2154-2161. Kyoto, Japan.

Goulette, F., Nashashibi, F., Ammoun, S., and Laurgeau, C. (2006). An integrated on-board laser range sensing system for On-the-Way City and Road Modelling. The ISPRS International Archives of Photogrammetry, Remote Sensing and Spatial Information Sciences, XXXVI-1:1-6.

Hernández, J. and Marcotegui, B. (2009). Filtering of artifacts and pavement segmentation from mobile LiDAR data. The ISPRS International Archives of the Photogrammetry, Remote Sensing and Spatial Information Sciences, XXXVIII-3/W8:329-333.

IGN (2013). IGN - Geospatial and Terrestrial Imagery. IGN French National Mapping Agency. http:// isprs.ign.fr/home Len.htm (Last accessed: October 28, 2013).

ISPRS (2013). The ISPRS data set collection. ISPRS International Society for Photogrammetry and Remote Sensing. http://www.isprs.org/data/ (Last accessed: October 28, 2013).

Munoz, D., Bagnell, J. A., Vandapel, N., and Herbert, M. (2009). Contextual Classification with Functional Max-Margin Markov Networks. In IEEE Computer Society Conference on Computer Vision and Pattern Recognition (CVPR).

Nüchter, A. and Lingemann, K. (2011). Robotic 3D Scan Repository. Jacobs University Bremen $\mathrm{gGmbH}$ and University of Osnabrück. http://kos.informatik. uni-osnabrueck.de/3Dscans/ (Last accessed: December 16, 2013).

Serna, A. and Marcotegui, B. (2013a). Attribute controlled reconstruction and adaptive mathematical morphology. In 2013 International Symposium on Mathematical Morphology (ISMM), pages 207-218, Uppsala, Sweden.

Serna, A. and Marcotegui, B. (2013b). Detection, segmentation and classification of 3D urban objects using mathematical morphology and supervised learning. $\{$ ISPRS $\}$ Journal of Photogrammetry and Remote Sensing, 0(0):1-. (Accepted).

Serna, A. and Marcotegui, B. (2013c). Urban accessibility diagnosis from mobile laser scanning data. ISPRS Journal of Photogrammetry Remote Sensing, 84:2332.

Velodyne (2012). The HDL-32E Velodyne LiDAR sensor. Velodyne Lidar 2012. http://velodynelidar. com/lidar/hdlproducts/hdl32e.aspx (Last accessed: December 16, 2013).

Zhou, L. and Vosselman, G. (2012). Mapping curbstones in airborne and mobile laser scanning data. International Journal of Applied Earth Observation and Geoinformation, 18(1):293-304. 